\title{
Eternal Tumor Virus (ETV): A new development in the treatment of benign and malignant tumors (cancer)
}

\author{
Habiba El Sayed \\ Mohammed Rashad Mohammed \\ DOI: 10.31364/SCIRJ/v8.i11.2020.P1120819 \\ http://dx.doi.org/10.31364/SCIRJ/v8.i11.2020.P1120819
}

\begin{abstract}
The relationship between cancer regression and viruses has long been theorized about, with case reports of the observed regression in cervical cancer, Burkitt lymphoma and Hodgkin's lymphoma, following immunization or infection with an unrelated virus at the beginning of the twentieth century. Efforts to treat cancer through immunization or virotherapy (deliberate infection with a virus) began in the mid-twentieth century. Since the technology to create a custom virus did not exist, all early efforts focused on creating naturally occurring tumor-resolving viruses. During the 1960s, promising research was involving the use of poliovirus, adenovirus, Coxsackie virus, enterovirus Riga virus and others. Early complications were occasional cases of uncontrolled infection, resulting in significant morbidity and mortality rates; The very frequent development of the immune response, while it is not harmful to the patient, led to the destruction of the virus, and thus prevented it from destroying the tumor. Therefore, my study followed up and searched for the best types of viruses that could not affect in any way on the human being because there is a fast and guaranteed solution to treat various tumors. Its forms are either a benign or malignant tumor (cancer).
\end{abstract}

Index Terms - Brain, Viruses, Tumors, Genetic testing.

Introduction:

Brain cancer, or an intracranial tumor, occurs when abnormal cells form inside the brain. There are two main types of tumors: a malignant (cancerous) tumor and a benign tumor.

Carcinomas are divided into primary tumors that start inside the brain, or secondary tumors that spread from another place to settle inside the brain, or what is known as metastasis in the brain. In this article we will deal with primary tumors. All types of tumors may produce symptoms that vary according to the location of the tumor in the brain. Symptoms include: headache, nerve convulsions, visual problems, vomiting, and mental disorders. Traditionally, headaches are worst in the morning, and this headache is eased when vomiting. More specific problems include: difficulty walking, speech and feeling. As the disease progresses, the patient may reach a subconscious state (coma).

The cause of most brain tumors is unknown. Risk factors include hereditary genetic factors known as neurofibromatosis, as well as exposure to industrial chemicals such as vinyl chloride, Epstein-Barr virus and ionizing radiation. There have been some concerns about mobile phone radiation, but they have not been scientifically proven. The most common types of primary tumors in adults are: meningiomas (usually a benign tumor), and astrocytomas such as glioblastoma. In children, the most common type is malignant myeloma. Diagnosis is usually by a medical examination in addition to a CT or magnetic resonance imaging. This is usually proven by a biopsy. Based on research results; Tumors are divided into different degrees of severity.

Treatment may include a combination of surgery, radiotherapy and chemotherapy. If seizures occur, seizure medications may be needed. Dexamethasone and furosemide may be used to reduce swelling around the cancer. Some tumors grow gradually, which only requires monitoring, and may not need any other intervention. Treatments that use a person's immune system are currently being

www.scirj.org

(C) 2020, Scientific Research Journal

http://dx.doi.org/10.31364/SCIRJ/v8.i11.2020.P1120819

This publication is licensed under Creative Commons Attribution CC BY. 
studied. Results vary greatly depending on the type of tumor and how far it has spread at diagnosis. Glioblastomas usually have poor results, while meningiomas usually have good results. The average five-year survival rate for brain cancer patients in the United States is $33 \%$.

Secondary or metastatic brain tumors are more common than primary brain tumors, and approximately half of metastases are from lung cancer. Primary brain tumors occur in about 250,000 people annually worldwide, making up less than $2 \%$ of cancers. In children under the age of 15, brain tumors are second only to acute lymphocytic leukemia as the cause of cancer. In Australia, the average economic cost of a case of brain cancer is $\$ 1.9$ million, more than any type of tumor.

\section{Problem to be solved:}

Or the following solutions that greatly affect people and are difficult to provide and do not provide all the ideal solutions

Surgery: Surgery is one of the main and effective cancer treatment methods against many different types of cancer, and it is the oldest treatment method that is being followed so far, and the choice of resorting to surgical treatment to get rid of cancer depends on a number of different factors such as the general health of the affected person, the type of cancer, The size of the tumor and its transfer to other parts of the body, and the location of the injury, and surgical treatment may be sufficient to eliminate cancer in some cases, or other treatment methods may be followed in conjunction with surgery, and in fact, early detection of cancer contributes to the success of surgery due to containment Cancer is often in a specific location in the body in its early stages. Chemotherapy: Chemotherapy or chemotherapy can be defined as one of the methods of treating cancer through the use of some cytotoxic drugs, which help eliminate cancer cells or reduce their ability to grow and spread, and chemotherapy is a systemic treatment because of its transmission through the blood And targeting all cells that rapidly divide and grow in the body, and in some cases the drug may be delivered to the cancerous tumor directly, and the treatment is local in this case, and in the context of the conversation the method of delivery of chemotherapy drugs, the duration of treatment, and the period between treatment doses varies according to the condition of the affected person and the type of drug The user may receive a single dose per day, or a dose every several weeks, and the affected person may be given periods of rest between doses of the drug, and often the chemotherapy dose is obtained in the hospital, but some types of treatment can be obtained in At home, chemotherapy drugs are available in several different pharmaceutical forms, such as oral pills, capsules, liquid medicines, intravenous infusions, and Topical medications.

Radiation therapy: Radiation therapy is defined as using high-energy radiation to target and destroy the genetic material of cancer cells to inhibit their ability to divide and grow, and it is one of the most effective types of treatments used to eliminate cancer after surgery depending on the condition of the affected person. The use of radiation therapy in the early stages of the disease or in the advanced stages in which the tumor has spread to other areas of the body.

Targeted therapy: It is a drug that targets specific and precise areas in cancer cells or one of the factors that contribute to their growth. It may target specific proteins or genes within cancer cells, or within cells that contribute to their growth and spread, such as blood vessel cells Feeding cancerous tumors.

Immunotherapy: Immunotherapy is a form of cancer treatment that aims to boost the body's immunity to eliminate cancer cells or prevent or control their formation. This is done by alerting the immune system to the presence of cancer cells, or by strengthening immunity and supplying The immune system has some elements that increase its defense capacity.

Stem cell transplants: Stem cell transplants depend on transplanting cord blood or bone marrow transplants for the affected person to help eliminate cancer, and this type of treatment is often followed in the treatment of cancers that affect the blood, Or the immune system such as lymphoma, leukemia, and multiple myeloma, and this type of treatment also includes removing cells affected by chemotherapy and radiation, then laboratory treatment and re-implantation in the body

Hormonal therapy: Hormonal therapy for cancer can be defined as treatment that eliminates or inhibits some types of hormones, or obtains them to help reduce or limit the speed of growth of cancerous tumors, and like other types of cancer treatments, hormonal therapy may be used alone It may be used to treat cancer, or it may be combined with other types of treatment, and it may be used to

www.scirj.org

(C) 2020, Scientific Research Journal

http://dx.doi.org/10.31364/SCIRJ/v8.i11.2020.P1120819

This publication is licensed under Creative Commons Attribution CC BY. 
increase the efficiency of some types of treatment as well, such as preventing recurrence and spread of cancer, or shrinking the size of the tumor before using other treatment methods.

\section{Research:}

In the beginning, I searched for previous solutions to problems, tumors of all kinds, whether benign or malignant (cancerous), and I found the use of the virus in Germany, the cowpox virus but when the search was completed due to problems, I searched a lot and found that the cowpox virus causes problems for humans, and after a lot of research, I found that the smallpox virus Goats and sheep unlike the cowpox virus does not affect any part of the human being.

So it was the beginning of the solution, and with other details, it was found that using this virus, which did not work in Germany for their use of the wrong virus, was the result of mice the disappearance of tumors and the occurrence of problems in the body, but there it became immunity Because no tumor came again, and by linking the details.

I came to the conclusion that why they not thought of using an alternative virus and it turned out that this was because of the recent tragic accident, which is the Corona virus. Two different solutions are the tumor and the work of a permanent vaccine to ensure that it does not become infected again, so it can be used in another way by using it for future generations at birth and after that, we test the genes with the Laura This is to check the presence of any part in the genes or not, and the virus is injected protection, and a permanent vaccine for them from any cancer or any tumor.

And that we will solve the problem of tumors present in the current adults and control the future generations and not have any injury whatsoever and when looking in particular for problems Brain tumors, we find a problem in the presence of a blood vessel that is difficult to reach for the tumor through surgery or when performing laser operations again.

\section{Other solution already tried:}

(experimental cancer vaccine developed) figure.1

The results, published in the New England Journal of Medicine, are the latest developments in an experimental cancer vaccine developed by the Duke Cancer Institute in North Carolina, USA, for patients with glioblastoma multiforme (glioblastoma), a fierce type of brain cancer.

www.scirj.org

(C) 2020, Scientific Research Journal

http://dx.doi.org/10.31364/SCIRJ/v8.i11.2020.P1120819

This publication is licensed under Creative Commons Attribution CC BY. 


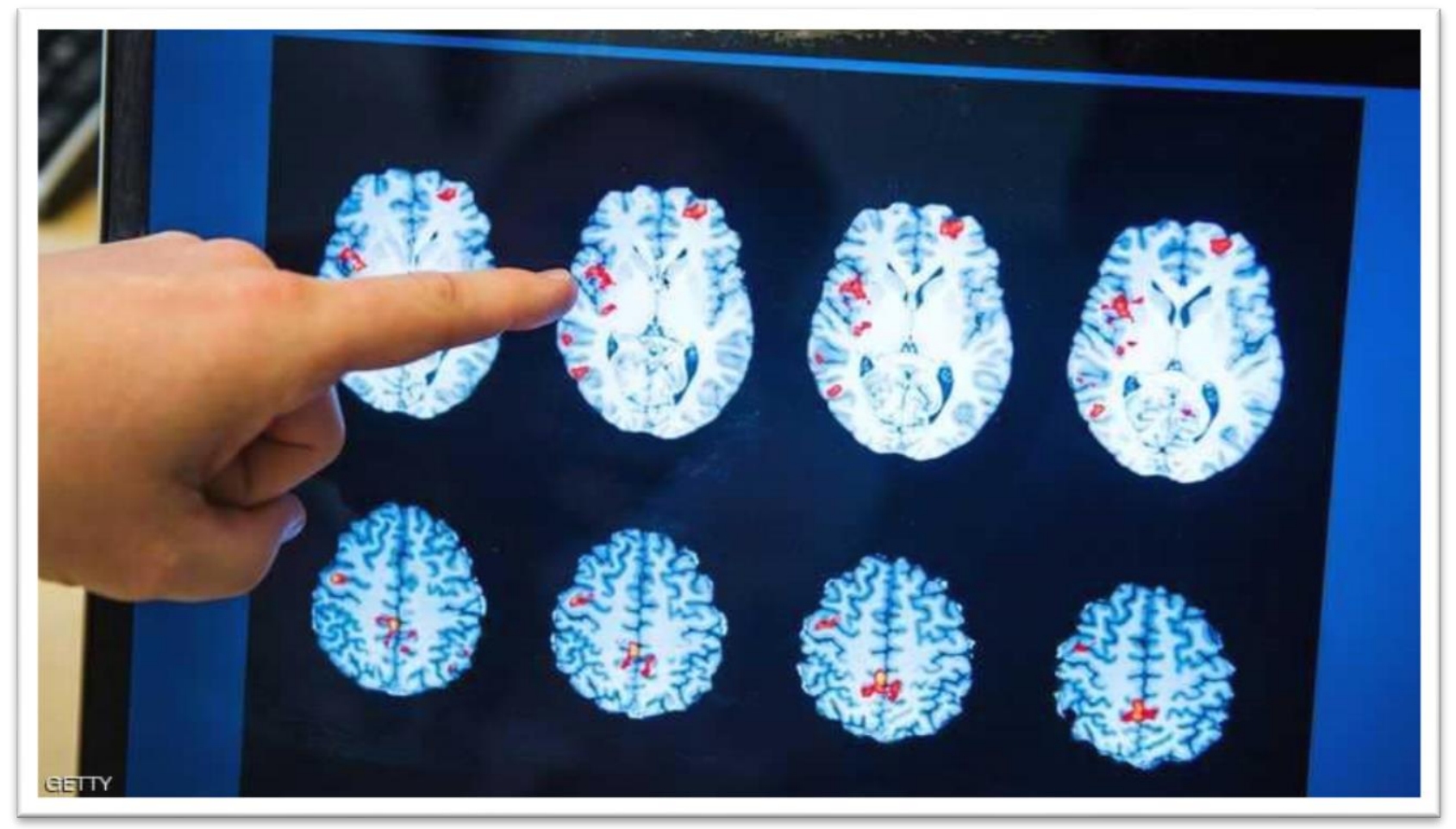

Figure.1

And patients with "glioblastoma" after the disease recurrence to attack them live for an average of 12 months, and treatment of this type of cancer usually includes chemotherapy, radiation, and targeted therapies.

The experimental vaccine includes a form of the poliovirus after genetic modifications, as it is injected into the tumor in the brain through a surgical catheter, to work to sharpen the immune system targeting the tumor cells.

The aim of the first experimental phase of the vaccine was to reach a safe dose, as the progress of 61 patients treated with the genetically modified poliovirus vaccine was compared with the records of other patients who received conventional treatment.

Some patients showed a great response to the vaccine, as two of them remained alive for at least 69 months, but most of them did not benefit from it, and side effects related to the vaccine appeared in about 69 percent of them.

"As with many immunotherapy treatments, some patients do not seem to respond for one reason or another, but when they do they stay alive for a long time," said Annick Dehgarden, one of the researchers involved in the study, in a statement. Scientists are currently working on the second experimental phase of the vaccine, to develop it and find out why some patients do not respond to it. 


\section{Solution (The theory is based on previous results):}

\section{Materials:}

1- Virus (smallpox virus of sheep and goats)

2- A specialized medical injection to insert the virus

\section{3- Genetic testing}

The solution is by the first way:

This solution is for newborns of future generations to permanently end all types of tumors or what is called cancer

At first, we do a genetic test, but at first, we must know why

Let's make an age if there are any mutations in the genes or not, and in both cases, it will be used as a permanent cancer vaccine (malignant tumors)

Second, we inject the virus in the body, whether there are problems with the genes or not, and thus the person is completely immune from infection with any tumor, whether it is malignant or benign

So that the virus targets the proteins responsible for the excessive division activity in the glial tumor cells in the brain. The virus is injected directly into the tumor, the virus attaches to the tumor cells, penetrates inside them, and destroys their ability to divide, leaving the immune system with the remaining task, which is dealing with the basic tumor cells, which lose their ability to reproduce, and ultimately die.

The designer virus is able to kill cancer cells, while not harming normal cells, something that current treatments lack. The current strategies depend on several options, the most important of which is the surgical option, in which doctors remove the tumor completely. It is an unacceptable and feasible option when these tumors are in sensitive places or brain centers responsible for the senses or movement.

In this case, doctors resort to using the second option, which is chemotherapy, and the effectiveness of chemical treatments is limited due to the presence of the blood-brain barrier, which prevents treatments from reaching deep into the brain, in addition to the very violent side effects of this type of medicine. As for radiation therapy, its ability is very limited in dealing with infectious tumors in

www.scirj.org

(C) 2020, Scientific Research Journal

http://dx.doi.org/10.31364/SCIRJ/v8.i11.2020.P1120819

This publication is licensed under Creative Commons Attribution CC BY. 
children's brains, which means that the use of strategies based on direct injection of viruses into tumors may be beneficial in the case of treating gliomas in children.

The second way:

If the person is infected and is very old, we will inject this virus in the place of the tumor and it will end the virus on the tumor and form a white substance on the tumor, which indicates the activation of the immune system as well, which helps in getting rid of the tumor

The third way: (especially related to brain tumors):

The problem of some uses of lasers or chemicals to eliminate tumors of all kinds is that there is a barrier blood vessel surrounding the brain, and we can open it using Gamma Knife, which is scientifically proven open for 4 weeks, and through which the virus can be injected to get rid of tumors and that is for adult people if it is not discovered It was reported early or was discovered in the usual natural way, and not by the first solution

\section{Conclusion:}

A group of doctors discovered a new way to treat tumors,

This method relies on injecting the patient with a genetically modified virus that attacks brain tumors or any type of tumor by creating a type of infection in them that prevents them from reproducing, limits their activity, and stops the process of replication, which gives the immune system the necessary opportunity to eliminate the original tumor in the brains of children.

According to the study published in the journal "Nature Communications", the researchers designed a virus capable of killing cancer cells, while not harming normal cells, but had side effects for selecting an inappropriate virus, and thus studies stopped by 2020 for the spread of the Corona virus, and so I completed the rest of the studies to find a type. Of viruses, which is Virus (smallpox virus of sheep and goats) for treatment and have no effect on humans or cause any problems, so the process will be a success due to previous experiences and the presence of a solution to the problems that were issued and with the inability of the materials to carry out actual experiments.

\section{Literature Cited:}

1- (n.d.). Retrieved August 14, 2020, from http://www.bafree.net/alhisn/archive/index.php/t-148168-p-2.html

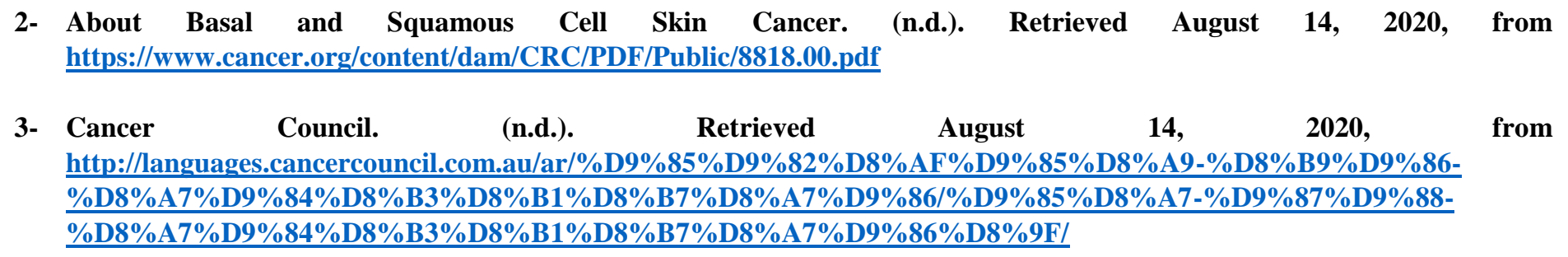

www.scirj.org

(C) 2020, Scientific Research Journal

http://dx.doi.org/10.31364/SCIRJ/v8.i11.2020.P1120819

This publication is licensed under Creative Commons Attribution CC BY. 
4- (الأورام السرطاوية. (2018, December 12). Retrieved August 14, 2020, from https://www.mayoclinic.org/ar/diseasesconditions/carcinoid-tumors/symptoms-causes/syc-20351039

5- Genetic testing. (2020, April 14). Retrieved August 14, 2020, from https://www.mayoclinic.org/tests-procedures/genetictesting/about/pac-20384827?fbclid=IwAR3wpC12gx7BFead1b2TBGuIlxXtaHtj3scYrYgyLHN0ROLze6VrCAYCKdII

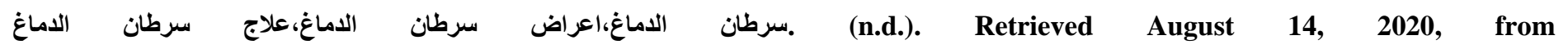
https://www.webteb.com/cancer/diseases/\% D8\%B3\%D8\%B1\%D8\%B7\%D8\%A7\% D9\%86-

\%D8\%A7\% D9\%84\%D8\% AF\%D9\%85\%D8\%A7\%D8\%BA 